\title{
Correction to: User Preference Prediction in a Set of Photos Based on Neural Aggregation Network
}

Kirill V. Demochkin and Andrey V. Savchenko

\section{Correction to:}

Chapter "User Preference Prediction in a Set of Photos Based on Neural Aggregation Network" in:

I. Bychkov et al. (eds.), Network Algorithms, Data Mining, and Applications, Springer Proceedings in Mathematics \& Statistics 315, https://doi.org/10.1007/978-3-030-37157-9_8

In the original version of this chapter, the title of Chapter 8 has been changed from "Visual Product Recommendation Using Neural Aggregation Network and Context Gating" to "User Preference Prediction in a Set of Photos Based on Neural Aggregation Network". The chapter and book have been updated with the changes. 